\title{
Compiler-Driven Data Layout Transformation for Heterogeneous Platforms
}

\author{
Deepak Majeti ${ }^{1}$, Rajkishore Barik ${ }^{2}$, Jisheng Zhao ${ }^{1}$, \\ Max Grossman ${ }^{1}$, and Vivek Sarkar ${ }^{1}$ \\ 1 Rice University \\ ${ }^{2}$ Intel Labs
}

\begin{abstract}
Modern heterogeneous systems comprise of CPU cores, GPU cores, and in some cases, accelerator cores. Each of these computational cores have very different memory hierarchies, making it challenging to efficiently map the data structures of an application to these memory hierarchies automatically. In this paper, we present a compiler-driven data layout transformation framework for heterogeneous platforms. We integrate our data layout framework with the data parallel construct, forasync, of Habanero-C and enable the same source code to be compiled with different data layouts for various architectures. The programmer or an auto-tuner specifies a schema of the data layout. Our compiler infrastructure generates efficient code for different architectures based on the meta information provided in the schema. Our experimental results show significant benefits from the compiler-driven data layout transformation, and demonstrate that the best data layout for a program varies with different heterogenous platforms.
\end{abstract}

\section{Introduction}

Recent hardware trends have seen the adoption of heterogeneous systems consisting of standard processor cores, graphics processing cores, and accelerator cores. While the memory hierarchy of standard CPU cores consist of L1, L2 and L3 caches, recent discrete GPU cores have also been embedded with their own L1, and L2 caches. Integrated GPU cores, on the other hand, share the same physical memory with the CPU while using a private L3 cache for the GPU cores. With such differing memory hierarchies within the same system, determining the best data layout can be challenging since the optimal layout for a computational kernel depends on whether the kernel executes on a CPU core, a discrete GPU, or on an integrated GPU (along with other factors). Additionally, the GPU memory performance is impacted by the number of coalesced memory accesses and control-flow divergence, whereas CPU memory performance is impacted by factors such as false sharing, prefetching and data reuse. This implies that the changes in data layout can have major impact on CPU vs. GPU core performance. In general, the programmer has to write different versions of CPU and GPU kernels for different architectures and has to select optimal memory layouts for each data structure. This places a severe constraint on code portability. At the same time, performing these layout transformations automatically for a wide-range of applications including irregular applications is a daunting task. 
CUDA and OpenCL are the two primary languages targeting the heterogeneous systems for GPGPU programming. OpenCL can also be used for targeting CPU cores. Many high-level programming models have also evolved in the last few years that deal with heterogeneity [1421 13 22 6 12]. Choosing a language involves trade-offs between programmer productivity and performance. However, none of these languages provide mechanisms to specify the data layout. Some recent work 201019 provide a library based mechanism, but are limited in scope. For example, Kunkel et.al. 24] emphasize the need for a data layout abstraction. Recently, Wu et al [23] have proved that finding the optimal data layout to maximize the number of coalesced accesses on a GPU is NP-complete. Thus, manually writing high performance portable programs or automatically generating efficient code via optimizing compilers without any domain knowledge is challenging given the proliferation of device technologies on heterogeneous architectures and their differing memory hierarchies. We believe that a compiler-driven data layout transformation framework can help bridge this gap.

In this paper, we present a compiler driven meta-data framework that allows both programmers and tuning experts to specify architecture specific and domain specific information for parallel-for loops of a program. A meta-data file is created for an application and is populated with entries on the data layout to be used for a device on the heterogeneous system. The data layout we focus on in this paper include structure-of-array (SOA), array-of-structure (AOS) and intermediate structure-of-array-of-structures (SOAOS). Any high level language which has parallel-for loops can be extended to accommodate the metadata framework. In our work, we target the data-parallel forasync construct in Habanero- $\mathrm{C}[2$ and integrate our meta-data framework with the Habanero- $\mathrm{C}$ compiler and runtime. Habanero- $\mathrm{C}$ compiles the forasync construct, to generate OpenCL device and host code for the target heterogenous architecture. The meta-data information is very useful in guiding the compiler optimization passes for the generation of efficient code for a device.

Our paper makes the following contributions:

- A meta-data framework that allows both the programmer and the tuning expert to specify the underlying architecture and domain specific knowledges for parallel-for loops;

- A compiler and runtime framework to automatically generate efficient code based on the meta-data information. We currently focus on AOS, SOA and SOAOS data layouts in our compiler;

- An experimental evaluation of our system using a wide variety of heterogeneous architectures which shows the impact of data layout on 5 distinct applications. On an average, the data layout transformation alone impacted the performance by $7.33 \times$ (up to $27.11 \times$ ) on AMD 4-core A10-5880K CPU, $2.84 \times$ (up to $5.57 \times$ ) on AMD Radeon integrated GPU, $8.32 \times$ (up to $29.5 \times$ ) on NVIDIA Tesla M2050 GPU, $2.19 \times$ (up to $5.32 \times$ ) on Intel 12-core X5660 CPU and $1.9 \times$ (up to $3.89 \times$ ) on Intel integrated i7-3770 GPU.

The rest of this paper is organized as follows. Section 2 presents our meta-data framework. Section 3 discusses the details of our compiler code generation and runtime. Section 4 presents the experimental results on a wide variety of processors. Related work is discussed in Section 5, and finally, Section [6 concludes. 


\section{Programming Model}

Our meta-data framework is built on top of Habanero-C (HC) compiler and runtime infrastructure 9. The details of the parallel constructs supported by $\mathrm{HC}$ can be found at [2]. Our paper focuses on the data parallel forasync construct 1 . The syntax of the forasync construct is as follows.

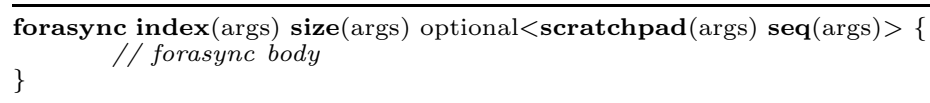

The semantics of the forasync construct is similar to a program loop which exhibits parallel_for parallelism. The index clause is used to specify the loop iterators. The number of variables in the index clause gives the dimentionality of the loop. The size clause specifies the number of iterations of the loop in each dimension. There are 2 optional clauses, scratchpad and seq clause. The HC language model takes advantage of the different memory regions available on most GPU hardwares with the help of the scratchpad and seq.

For each host or the device on a heterogeneous system, it is possible to specify the desired data layout for array-based or structure-based data structures of a given forasync loop. The data layouts that we focus on are: (1) AOS: arrayof-structure; (2) SOA: structure-of-array and (3) SOAOS: structure-of-array-ofstructures. Our compiler (described in Section 3) with the help of the meta-data file is able to transform HC code to one of the SOA, AOS and SOAOS layouts.

The grammar for the meta-data and an example is shown in Figure 11. The

\begin{tabular}{|c|c|}
\hline $\begin{array}{l}\text { arch_name }->\text { Arch name meta_data } \\
\text { meta_data }->\text { (struct_def) } * \text { (scratchpad_def }) * \\
\text { struct_def }->\text { Struct name (field_def) } * \\
\text { scratchpad_def }->\text { Scratchpad name } \\
\quad \text { (field_def tile_size line_num)* } \\
\text { field_def }->\text { Field type name length } \\
\text { type }=\text { fp } \mid \text { dp } \mid \text { ip } \\
\text { length }->\text { (digit) } * \\
\text { tile_size }->\text { (digit) } * \\
\text { line_num }->(\text { digit }) * \\
\text { name }=(\text { letter })(\text { letter } \mid \text { digit }) * \\
\text { letter }->-|\mathrm{A}| \mathrm{B}|\mathrm{C}| \text {. }|\mathrm{Z}| \mathrm{a}|\mathrm{b}| \mathrm{c}|\ldots| \mathrm{z} \mid \\
\text { digit }->1|2| 3|4| 5|6| 7|8| 9 \mid 0\end{array}$ & \begin{tabular}{|l|} 
Arch Intel_GPU \\
Struct bodypos \\
Field fp posx Field fp posy Field fp posz \\
Struct bodyacc \\
Field fp accx Field fp accy Field fp accz \\
Scratchpad local Field fp posx 25664 \\
Scratchpad local Field fp posy 25664 \\
Scratchpad local Field fp posz 25664 \\
Arch AMD_GPU \\
Struct bodypos \\
Field fp posx Field fp posy Field fp posz \\
Field fp accx Field fp accy Field fp accz \\
Scratchpad local Field fp accx 102464
\end{tabular} \\
\hline
\end{tabular}

Fig. 1. Meta-data Grammar (left) and meta-data file Example (right)

meta-data file consists of a set architecture specific optimization information. The architectural details consist of the data layout information and scratchpad memory allocation information for a given program. Each struct definition has a label Struct, a name for the struct and a set of fields. Each field in turn has a label Field, the type of the field and the name of the field. The type of fields can be $f p$ : a pointer to an array of float values, $d p$ : a pointer to an array of double values or $i p$ : a pointer to an array of integer values. The scratchpad memory allocation information consists of a set of buffer descriptions. It begins with a label Scratchpad, the name of the special memory region, the field, the amount of data which must be cached and the line number of the forasync.

${ }^{1}$ Our framework is also applicable to other data-parallel programming languages with a parallel-for like construct. 


\section{Restrictions of our meta-data framework}

The user cannot alias the fields specified in the meta-data file. We plan to be resolve this issue with the help of an alias analysis. Another limitation in the programming model is that a variable name cannot be repeated in the whole program in different scopes. This limitation can be avoided by a clever variable renaming mechanism. Also, all fields in a struct must be of the same type. We currently do not support more complex data layouts such as AOSOA (Array-ofstructure-of-arrays). We leave this for future work.

\section{Implementation}

Our overall meta-data framework is shown in Figure 2, The application user writes a program in Habanero-C (HC) using the forasync construct. Followed by which, either the developer or the tuning expert specifies the meta-data information for the application. We extend the $\mathrm{HC}$ compiler infrastructure to perform data layout transformation based on the meta information;. The compiler pass is implemented in the ROSE source-to-source compiler framework [17. The HC compiler generates OpenCL code from the program with the specified data layout and the corresponding host code.

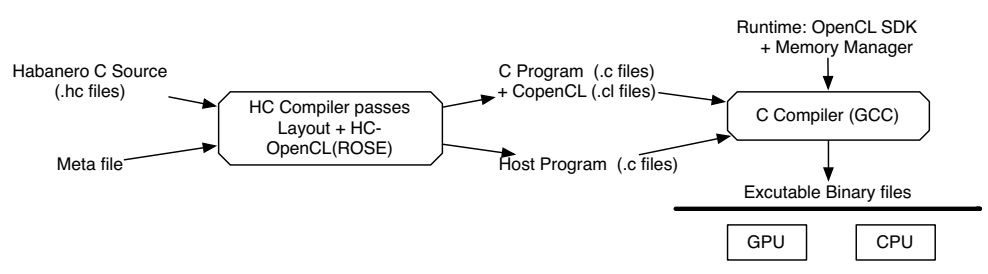

Fig. 2. Compilation Flow

\subsection{Data Layout Transformation}

The compiler pass first parses the specified meta-data file and it creates a metadata map for each architecture. The mapping is between the fields and the struct name they belong to. The mapping is done for each such struct meta information. If it finds any scratchpad meta information, it is recorded.

The data layout transformation (DLT) compiler pass generates the code based on the specified data layout in the meta-data file. It generates code which includes new struct definitions and the code that operates on it. Figure 3 shows the algorithm for transforming the program with a given data layout. DLT takes the input program and a meta-data file. createStructDefinitions(M) adds the struct definitions as specified in the meta-data file to the AST. These structs are defined only once in the global scope. The DLT pass then iterates over all the functions and performs the steps described in lines 4-7.

tryAddStructInstances ( $f$ ) analyzes the function parameters. If any of the parameter names appear in the meta file, an instance of the corresponding struct is declared in the function call. If we abstract the struct as a group of fields names, then one struct instance is declared per group. In next step, updateInst(I) 


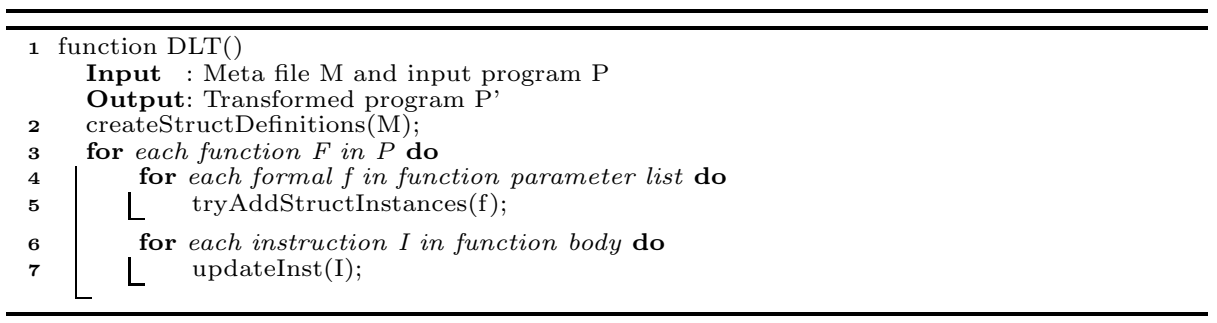

Fig. 3. Data Layout Transformation

checks all pointer or array references in the function body. If any of those reference are via any of the fields in the meta file, then the access is replaced with the corresponding struct instance.

An important factor here is that the type of the function in the original program remains the same. Keeping the function types intact will avoid rewriting the direct and indirect calls to the function from sequential code.

\subsection{Memory Management}

In the $\mathrm{HC}$ programming model, the programmer allocates heap memory to the fields via standard malloc and calloc calls. We replace these calls with our specialized memory allocators. We name the allocators, hc_meta_malloc or hc_meta_calloc. The syntax of the allocators is shown in Figure 4.

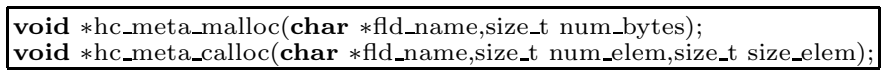

Fig. 4. Memory Allocators

$h c_{-} m e t a \_m a l l o c$ or $h c_{-} m e t a \_c a l l o c$ are wrappers around the standard malloc and calloc calls. The allocators also pass in the name of the field to the memory allocator. The field name is required by the memory manager and is explained as follows.

The memory manager handles the different layouts and also creates device buffers. The memory manager has two important components, the memory allocator and the layout handler. During the program initialization phase, the layout handler reads the meta file and creates a map of the data layout. The memory manager with the help of the field name, looks into the layout map and allocates the memory.

Figure 5] shows an example code generation of a single kernel with meta data information for an Intel architecture.

\section{Evaluation}

The goal of the experimental evaluation is to study the performance of different data structure layouts for various programs on multiple architectures. 


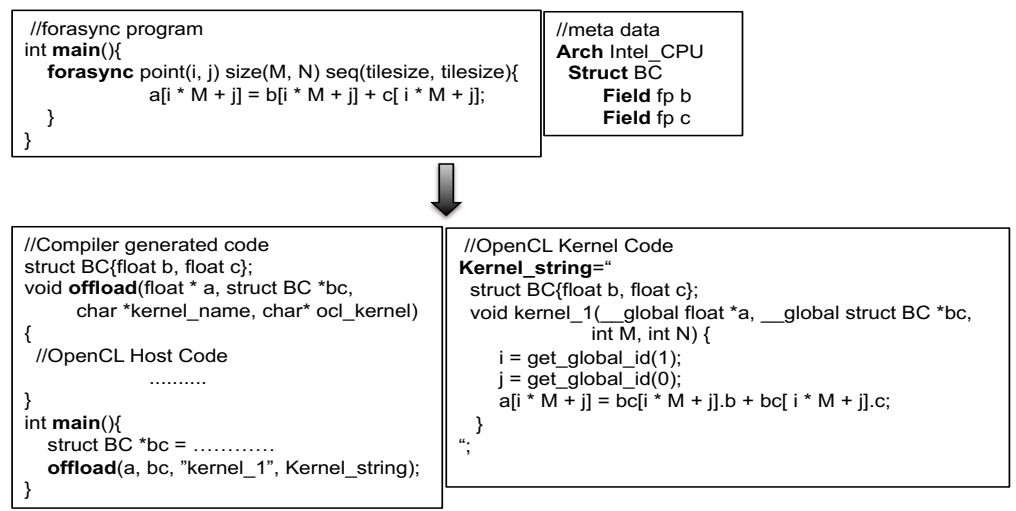

Fig. 5. Example of code generation using a meta-data file

Table 1. Benchmarks

\begin{tabular}{|c|c|c|c|c|}
\hline Name & Description & $\begin{array}{c}\text { Original } \\
\text { Layout }\end{array}$ & $\begin{array}{c}\text { Num of } \\
\text { Fields }\end{array}$ & Input \\
\hline NBody & N-Body Simulation & SOA & 7 & $32 \mathrm{~K}$ nodes \\
\hline Medical & Medical Image Registration & SOA & 6 & $256 \times 256 \times 256$ \\
\hline SRAD & Speckle Reducing Anisotropic Diffusion & SOA & 4 & $5020 \times 4580$ \\
\hline Seismic & Seismic Wave Simulation & $\mathrm{SOA}$ & 6 & $4096 \times 4096$ \\
\hline MRIQ & $\begin{array}{l}\text { Matrix Q Computation for 3D Magnetic Resonance } \\
\text { Image Reconstruction in Non-Cartesian Space. }\end{array}$ & $\mathrm{SOA}$ & 6 & $64 \times 64 \times 64$ \\
\hline
\end{tabular}

\subsection{Experimental Setup}

Table 1 describes the benchmarks used in this evaluation.

The N-Body particle simulation benchmark was written from scratch for this work. We focus on the compute intensive kernel which calculates the forces between the bodies.

The Medical Imaging benchmark includes kernels from a medical imaging pipeline used to analyze different types of medical images for defects or abnormalities [15]. This application consists of three main phases: denoising, registration, and segmentation. For our evaluation, we focus on the most computationally significant kernel of the three, registration.

The SRAD benchmark from the Rodinia benchmark suite [11] is also used. SRAD is used to "remove locally correlated noise" in "ultrasonic and radar imaging applications based on partial differential equations" [18].

The Seismic benchmark suite was created based on the example included in the Intel TBB benchmark suite [4]. Seismic simulates the propagation of waves during seismic activity.

The MRIQ benchmark from the Parboil benchmark suite 7] computes a $Q$ matrix. The $Q$ matrix represents the scanner configuration used in a $3 \mathrm{D}$ magnetic resonance image reconstruction algorithm in non-Cartesian space. The MRIQ code has been converted to SOA layout by hand.

Table 2 shows the different meta-data files used for each benchmark. Since the default layout is SOA, there is no need of a meta file. All OpenCL kernels, glue 
Table 2. Application meta-data files

\begin{tabular}{|c|c|c|}
\hline Application & AOS & SOAOS \\
\hline NBody & $\begin{array}{l}\text { Struct body Field fp posx Field fp posy Field fp posz } \\
\text { Field } f p \text { acc Field } f p \text { accy Field } f p \text { accz }\end{array}$ & $\begin{array}{l}\text { Struct pos Field fp posx Field fp posy Field fp posz } \\
\text { Struct acc Field fp accx Field fp accy Field fp accz }\end{array}$ \\
\hline Seismic & $\begin{array}{r}\text { Struct params Field fp S Field fp T Field fp V } \\
\text { Field fp D Field fp L Field fp M }\end{array}$ & N.A \\
\hline SRAD & $\begin{array}{l}\text { Struct direction Field fo N Field fo S Field fo } E \\
\text { Field fp W }\end{array}$ & $\begin{array}{l}\text { Struct direction1 Field fp N Field fp S } \\
\text { Struct direction2 Field fp E Field fp W }\end{array}$ \\
\hline Medical & $\begin{array}{l}\text { Struct disp Field fp U1 Field fp U2 Field fp U3 } \\
\text { Struct velocity Field fp V1 Field fp V2 Field fp V3 }\end{array}$ & Struct disp Field fp U1 Field fp U2 Field fp U3 \\
\hline MRIQ & $\begin{array}{l}\text { Struct body Field fp kx Field fp ky Field fp kz } \\
\text { Field fp phiMag }\end{array}$ & N.A \\
\hline
\end{tabular}

Table 3. Hardware architectures

\begin{tabular}{|l|l|l|l|l|l|l|l|}
\hline Vendor & Type & Model & Freq & Cores & Local Mem & L1\$ & L2\$ \\
\hline Intel & CPU & X5660 & $2.8 \mathrm{GHz}$ & $12(\mathrm{HT})$ & N.A & 192KB & $1.5 \mathrm{MB}$ \\
Intel & Integrated GPU & i7-3770U & $350 \mathrm{MHz}-1.15 \mathrm{GHz}$ & 14 & $64 \mathrm{~KB}$ (per half-slice) & N.A & N.A \\
NVIDIA & Discrete GPU & Tesla M2050 & $575 \mathrm{MHz}$ & 8 & $8 \times 48 \mathrm{~KB}$ & 16KB & $768 \mathrm{~KB}$ \\
AMD & CPU & A10-5800K & $1.4 \mathrm{GHz}$ & $4(\mathrm{HT})$ & N.A. & 16KB & $32 \mathrm{MB}$ \\
AMD & Integrated GPU & Radeon HD 7660 & $800 \mathrm{MHz}$ & 6 & $6 \times 32 \mathrm{~KB}$ & N.A & $4 \mathrm{MB}$ \\
\hline
\end{tabular}

code, and different layouts for each of these applications were generated from a $\mathrm{HC}$ array-based implementation.

Table 3 lists the hardware architectures used in our evaluation. We use a variety of CPU and GPU systems with differing memory hierarchies in order to demonstrate the benefit of our data layout transformation. The compiler used for the sequential versions of each application GCC 4.4.6 (with the flags -g -02). All OpenCL kernels were compiled with their default optimizations enabled. Intel GPU tests were run using the 2013 Release of the Intel OpenCL SDK [3]. Intel CPU tests were performed using 2011 Release of Intel OpenCL SDK, v1.5 3. NVIDIA GPU tests were performed using NVIDIA SDK v5.0 5]. AMD GPU and GPU tests were performed using AMD APP SDK v2.8 [1].

\subsection{CPU and GPU Performance}

Figure 6 contains results for all the benchmarks. We compare relative execution time for the various data layouts on different CPU and GPU platforms. For a given architecture, we normalize every layout with respect to the fastest executing layout. In this case, smaller bars imply better performance. Every column is stacked in 2 levels. The bottom level represents the fraction of total execution time spent in the kernel. This information is retrieved from the OpenCL API. The top stack represents the fraction of total execution time for the remaining execution. This includes communication and OpenCL initialization overheads. The top stack is negligible for Intel GPU. This is because the GPU is integrated one the same die as the CPU and there is no data copying overhead. NVIDIA GPU and AMD architectures show copying overheads. For all the workloads, the AMD CPU/GPU exhibit a large amount of overhead. On further inspection, we discovered that the majority of the overhead was due to significant time difference between OpenCL kernel enqueue and kernel execution. This could be an implementation error in AMD's OpenCL implementation. To analyze the performance differences, we could not find any tools which profile OpenCL code. We make the following analysis based on code and machine characteristics.

For the N-Body benchmark, we see that the SOA and AOS versions perform similarly on the CPU. Since the number of fields are less, all the loads in an 
iteration fit into the cache and consecutive iterations do not incur any penalty. The array layout performs better on GPUs because SOA layout helps in memory coalescing.

For the Seismic kernel, the SOA layout shows better performance on AMD CPU, whereas the AOS layout is better on Intel CPU. This can be attributed to the difference in cache associativity and sizes between AMD and Intel. On the GPU side, SOA performs well on all 3 GPU hardwares due to coalescing.

The SRAD kernel shows improved performance for the SOAOS layout relative to the SOA and AOS layout for most of the architectures. Surprisingly even on the GPU the AOS and SOAOS layouts performs better than the SOA layout. This is contrary to GPU best practices. The memory access functions in the SRAD kernel are non-affine and irregular. It is difficult for a compiler or programmer to analyze and determine the right layout. Our framework enables rapid prototyping and testing of different layouts for performance on multiple architectures.

MRIQ exhibits little or no variation across layouts. MRIQ is a compute bound kernel and the data structure layout will have little or no effect.

The medical image benchmark shows some interesting properties for different layouts. The AOS layout is better on the CPU whereas the SOA layout is better on the GPU. Medical image kernel is similar to a 3D Jacobi (stencil) computation. The stencil computation is performed separately on three input buffers and the results are written into corresponding output buffers. Keeping the input buffers in a single struct is helpful for the CPU. This is because when you load a point for one of the stencil, you automatically load the points for the other 2 stencils (multiple points fit in a cache line). The array layout would have caused 3 loads for the same point, one in each of the three stencils. On the GPU side, the array layout is better as expected.

Best practices generally dictate the use of array data layouts on GPUs due to improved coalescence of global memory accesses. However, our SRAD and MRIQ results contradict this knowledge. Our metadata framework enables rapid prototyping and optimization of different data layouts, allowing tuning experts to rapidly discover optimal layouts for complex and irregular applications. For the CPU the layout often depends upon the kernel features and memory access patterns. Our programming model can easily port such applications to different architectures.

\section{Related Work}

Recently, data layouts have been studied in the context of GPUs. DL 20] uses a mapping function and runtime library support to enable architecture specific data layouts. DL does in-place data marshaling on the GPU. Like DL, Dymaxion [10] proposes a set of index mapping functions which are used to optimize memory mappings, with data marshaling done on the CPU side. Sung et. al. 19] used techniques similar to DL to perform data layout transformations for structured grid applications. Their compiler automatically changes the order of n-dimensional array references to maximize memory access coalescing. With the help of micro-benchmarks, low latency strides and an optimal index map are discovered. This technique requires manual host code changes. The main disadvantage of the techniques listed in this paragraph is that the overhead of 


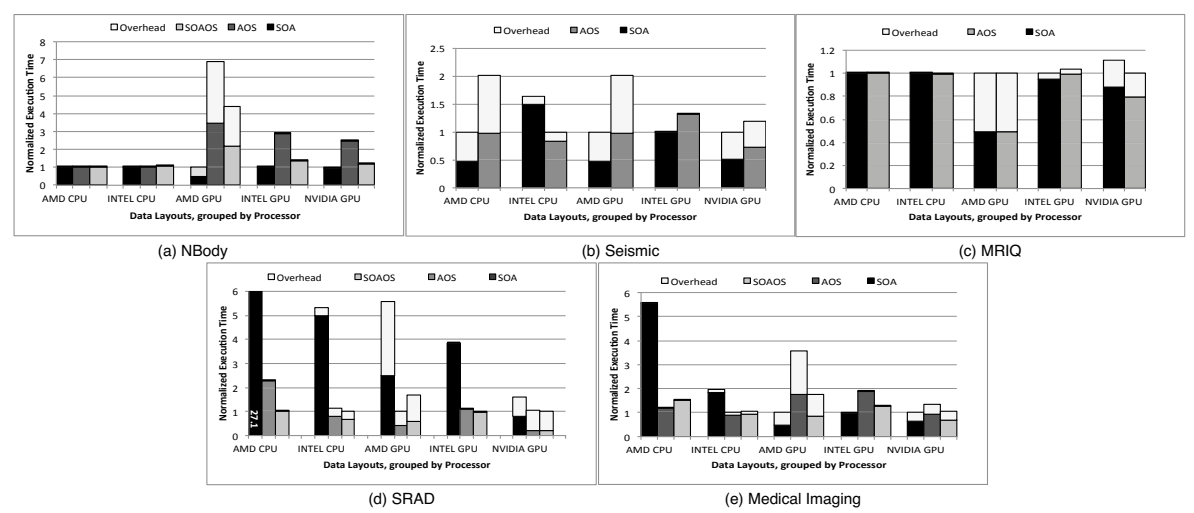

Fig. 6. GPU, CPU performance relative to the best layout (Lower the better). The darker bars show results for kernel execution time. The light bar shows results for communication and OpenCL initialization overhead.

runtime data marshaling can eliminate or reduce the performance benefits of optimal data layouts. Our compiler based approach does not incur this added overhead. The above runtime-based techniques are also either restricted to a class of applications (such as grid applications) or require manual changes by the programmer. Our compiler-based approach does not require any manual coding.

Ren et. al. 8 introduce an interpreter-based SIMDization mechanism that can parallelize the sequential programs that traverse on irregular data structures (e.g., binary decision tree and regular expression matching). To reduce memory latency, they chose different compacting policies based on layout including depth-first, breadth-first, and level-by-level. These policies improve memory access latencies for the irregular data structures. Compared to their work, our meta-data framework is not tied to any specific applications and that it can be easily extended to support the above data-layouts.

Liu et al [16] describe an automatic layout transformation that first divides arrays into blocks and then maps them to processing units with minimal overlap. Their approach does not perform any AOS to SOA transformation.

\section{Conclusions}

We present a compiler-driven data layout transformation that is applicable to any data parallel parallel_for programming model. The data layout transformation uses a "meta-file" approach which enables the same source code to be compiled with different layouts without involving the programmer worrying about it. Our experimental results show significant benefits from this approach and demonstrates that the best data layout for a given program can be different for CPU vs. GPU execution. With the growing users of GPUs in mainstream computing, it is important to have a tool like ours to understand the performance debugging. In future, we would like to develop a general heuristic to automatically perform data layout transformation that works across all platforms. 


\section{References}

1. Amd app sdk v2.8, http://developer .amd.com/tools/heterogeneous-Computing /amd-accelerated-parallel-processing-app-sdk

2. Habanero-c, https://wiki.rice.edu/confluence/display/HABANERO/ Habanero-C

3. Intel opencl sdk, http://software.intel.com/en-us/vcsource/tools/openclsdk

4. Intel thread building blocks, http://threadingbuildingblocks.org/

5. Nvidia sdk, https://developer.nvidia.com

6. Openacc, http://www.openacc-standard.org

7. Parboil benchmark suite, http://impact.crhc.illinois.edu/parboil.aspx

8. SIMD Parallelization of Applications that Traverse Irregular Data Structures. IEEE Computer Society (2013)

9. Chatterjee, et al.: Integrating asynchronous task parallelism with mpi. In: IPDPS $2013(2013)$

10. Che, et al.: Dymaxion: optimizing memory access patterns for heterogeneous systems. In: SC 2011, pp. 13:1-13:11. ACM, New York (2011)

11. Che, et al.: Rodinia: A benchmark suite for heterogeneous computing. In: ISWC 2009, pp. 44-54 (October 2009)

12. Microsoft Corporation. $\mathrm{C}++$ accelerated massive parallelism specification, http://msdn.microsoft.com/en-us/library/vstudio/hh265136.aspx

13. Cunningham, D., Bordawekar, R., Saraswat, V.: Gpu programming in a high level language: compiling x10 to cuda. In: X10 2011, pp. 8:1-8:10. ACM, New York (2011)

14. Dubach, et al.: Compiling a high-level language for gpus: (via language support for architectures and compilers). In: PLDI 2012, pp. 1-12. ACM, NY (2012)

15. Center for Domain Specific Computing. Cdsc research applications

16. Liu, et al.: Data layout optimization for gpgpu architectures. In: PPoPP 2013, pp. 283-284. ACM, NY (2013)

17. LLNL. Rose compiler infrastructure, http://rosecompiler.org/

18. Rodinia Benchmark Suite. Srad wiki page

19. Sung, et al.: Data layout transformation exploiting memory-level parallelism in structured grid many-core applications. In: PACT 2010, pp. 513-522. ACM, New York (2010)

20. Sung, I.-J., Liu, G.D., Hwu, W.-M.W.: Dl: A data layout transformation system for heterogeneous computing. In: InPar 2012, pp. 1-11 (May 2012)

21. Unat, D., Cai, X., Baden, S.B.: Mint: realizing cuda performance in $3 \mathrm{~d}$ stencil methods with annotated c. In: ICS 2011, pp. 214-224. ACM, New York (2011)

22. Vincent, C., Jisheng, Z., Jun, S., Vivek, S.: Habanero-java: the new adventures of old x10. In: PPPJ 2011 (2011)

23. $\mathrm{Wu}$, et al.: Complexity analysis and algorithm design for reorganizing data to minimize non-coalesced memory accesses on gpu. In: PPoPP 2013, pp. 57-68. ACM, New York (2013)

24. Zhang, Y., Sinclair II, M., Chien, A.A.: Improving performance portability in openCL programs. In: Kunkel, J.M., Ludwig, T., Meuer, H.W. (eds.) ISC 2013. LNCS, vol. 7905, pp. 136-150. Springer, Heidelberg (2013) 